\title{
ANÁLISE DE TRABALHO COLABORATIVO PARA GERAÇÃO DE MODELO BIM
}

\author{
Bruna Costa $^{(1)}$, Bruno de Brito ${ }^{(1)}$, Jessica Silva $^{(1)}$, Letícia Macedo $^{(1)}$, Vilca Santos ${ }^{(1)}$ \\ (1) SENAI CIMATEC, Salvador
}

\begin{abstract}
Resumo
A adoção da tecnologia BIM dissociada de metodologias de trabalho colaborativo implica no desenvolvimento de projetos cujos problemas se assemelham àqueles desenvolvidos em plataformas CAD: são fragmentados, sequenciais e com pouca interação entre os envolvidos. O objetivo deste artigo é analisar um processo de trabalho colaborativo utilizando um modelo compartilhado BIM. Como estudo de caso, foi escolhido um projeto residencial de múltiplos pavimentos, cujo modelo gerado seria usado como fonte de extração de documentação de projeto e quantitativos relevantes para a orçamentação. Antes de iniciar o trabalho, foram elaborados os documentos deliberantes: o Plano de Execução BIM e o caderno de diretrizes de modelagem, ambos para o LOD 300. O processo de trabalho colaborativo proposto foi avaliado segundo o tempo de modelagem de cada disciplina, o tipo e a frequência de comunicação entre os agentes envolvidos, a infraestrutura tecnológica e a qualidade da informação extraída do modelo. Como resultado, tem-se uma análise da modelagem colaborativa usando worksets visando entender quais são as possíveis melhorias de processo, os entraves e os desafios para essa metodologia de trabalho e de que forma ela pode retroalimentar os fluxos de projeto.
\end{abstract}

\section{Introdução}

Para que uma construção seja fidedigna ao modelo virtual e com exequibilidade garantida, é necessária a colaboração de profissionais de diversas disciplinas. No entanto, sem boas práticas de trabalho colaborativo até mesmo os processos BIM (Building Information Modeling) se tornam pouco assertivos como os processos em 2D. Tais processos são conhecidamente rudimentares. Segundo Coelho e Novaes [4], "a alteração de um projeto desenvolvido em CAD (2D e 3D) implica em diversas modificações manuais dos objetos representados". De acordo com Amor e Owen [1], um dos imperativos para a indústria AEC (Arquitetura, Engenharia e Construção) é que todos os processos sejam integrados. No entanto, mesmo após nove anos da publicação de seu artigo "Beyond BIM - It's Not the End of the Road!", vê-se ainda que estes métodos continuam refletindo a fragmentação da prática de projetos CAD. Para além da falta 
dos métodos de colaboração, os processos de projeto e modelagem também não possuem planejamento prévio, indicando uma falta de conhecimento dos conceitos apesar do aparente domínio das ferramentas BIM. Esse fator vai de encontro com a padronização e organização dos dados necessárias para permitir a colaboração entre os diversos agentes participantes do processo de projeto [4].

Em diversos países, o BIM já é exigido para aprovação e análise de projeto, o que exige não apenas a criação de um modelo tridmensional, mas sim a realização de um processo integrado. No entanto, existe uma tendência à utilização do BIM enquanto ferramenta [7]. Segundo Santos [9], "é de se esperar que para a adequada implementação do BIM sejam necessárias alterações nas formas de trabalho habituais praticadas nos empreendimentos da construção". O autor supracitado relata ainda que "a colaboração é fomentada e facilitada pelo processo BIM, mas requer mudanças de comportamentos dos vários agentes envolvidos no processo". O que se pretende é aperfeiçoar a resolução de problemas e executar edifícios mais eficientes e de alta qualidade.

O objetivo deste artigo é analisar um processo de modelagem BIM de modo a garantir a assertividade da informação e uma melhor colaboração entre os agentes envolvidos no desenvolvimento do projeto. Para a elaboração do trabalho fez-se necessário uma revisão da literatura, o estabelecimento dos fluxos de trabalhos e diretrizes de modelagem, e por fim, a análise dos resultados obtidos.

\section{Trabalho colaborativo em BIM}

Com a tecnologia BIM, um ou mais modelos virtuais precisos da edificação são construídos digitalmente e, quando finalizados, estes modelos gerados por computador apresentam uma geometria precisa com informações necessárias para auxiliar as atividades de construção, fabricação e compras que compõem a realização do edifício [5].

Essa modelagem pressupõe um trabalho integrado entre diversos profissionais, entretanto o contexto brasileiro ainda apresenta obstáculos a adoção do BIM. As principais barreiras para sua implementação estão relacionadas à resistência a mudanças, à falta de compreensão dos benefícios e potencialidades dessa tecnologia, além do frequente desinteresse pelo trabalho colaborativo [2].

Diante da complexidade do desenvolvimento de um modelo colaborativo novas abordagens são fundamentais para a elaboração dos projetos e para a troca de informações entre os envolvidos: "Processos padronizados e protocolos acordados são necessários para atribuir responsabilidades e conduzir as revisões e validações de projetos" [10].

Segundo a tabela de usos BIM desenvolvida pela Pennsylvania State University [6], compartilhar informações é um dos principais usos do BIM. Esta universidade também desenvolveu modelos de documentos que atendem aos padrões e procedimentos necessários a implantação do BIM nos projetos. Um dos temas abordados são os "procedimentos de colaboração", nele devem constar as estratégias de colaboração; as reuniões previstas em cada etapa do projeto e os envolvidos; os tipos de arquivos que serão compartilhados, por quem serão compartilhados, o software nativo etc; além da descrição do ambiente de trabalho interativo [8]. 


\section{Metodologia}

Para desenvolvimento do trabalho, foi utilizada a estratégia metodológica Estudo de Caso, que permite aos investigadores focar em um caso para ter uma visão holística e do mundo real [11]. Deste modo, o trabalho foi estruturado conforme a Figura 1.

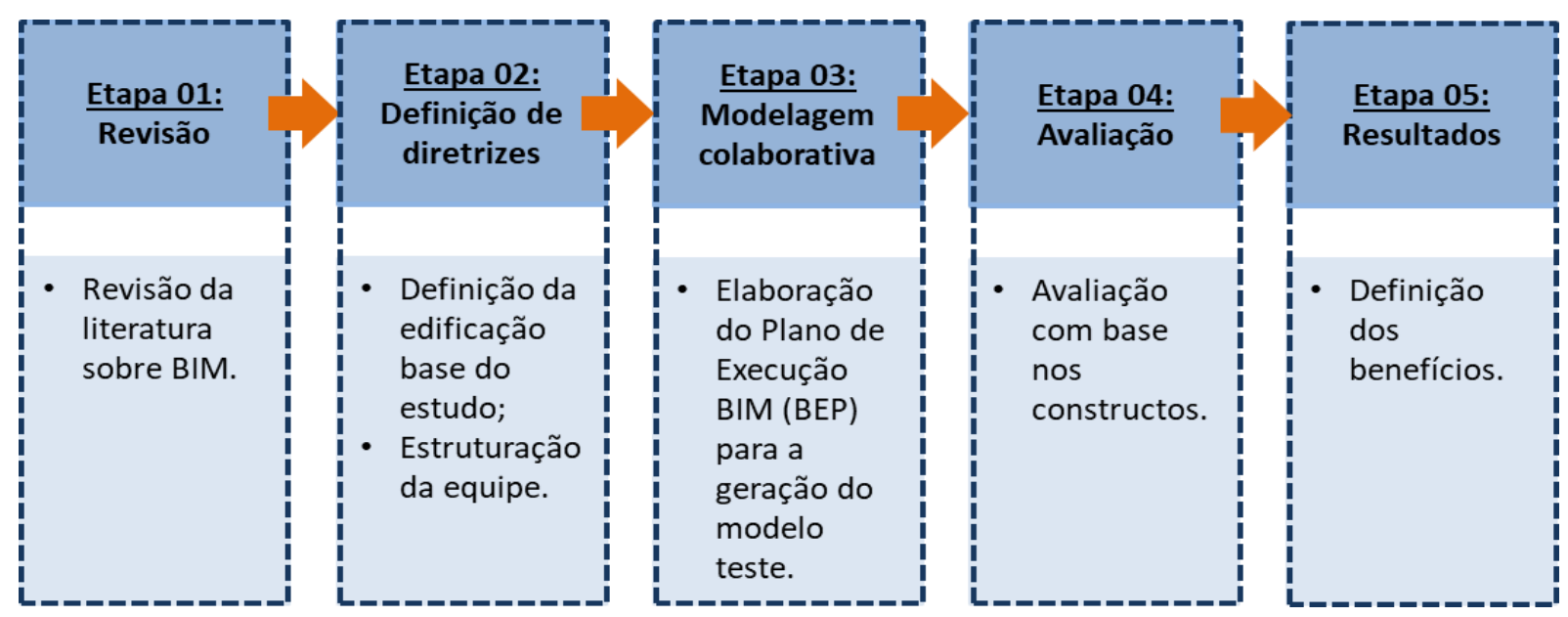

Figura 1: Processo de desenvolvimento do artigo.

Inicialmente, foi feita a revisão da literatura sobre trabalho colaborativo em BIM de modo entender o estado da arte do tema. Na Etapa 02, foi escolhido o projeto: uma residência com dois pavimentos e $294,16 \mathrm{~m}^{2}$, assim também como o ambiente de trabalho colaborativo. Este ambiente contou com acesso à internet (download de 20.8Mbps e upload de 4.26Mbps), computadores (Intel Core i7, com 8GB e 16GB de RAM) e servidor local (02 HDs DE 1T, PROCESSADOR XEON e 8GB MEMORIA) e conexão à rede via Wi-Fi. O software e sua versão, Autodesk Revit 2019, também foram definidos, possibilitando que a modelagem fosse feita em arquivo único utilizando os worksets.

A equipe foi dividida em um gerente BIM e três modeladores. Cada um dos atores ficou responsável por atividades específicas de modelagem das disciplinas de construção e configuração do modelo. As disciplinas contempladas pelo projeto foram: (1) projeto de arquitetura, (2) projeto de estrutura, (3) projeto hidrossanitário e (4) projeto elétrico.

A Etapa 03 se caracterizou como o momento de decisão sobre as questões intrínsecas à modelagem colaborativa. Neste momento, foi elaborado o Plano de Execução BIM, de modo a permitir que as disciplinas fossem modeladas em arquivo único e que todos os modeladores tivessem acesso aos mesmos objetos inteligentes além de configurações de modelagem e documentação. A avaliação foi feita com base no tempo de modelagem total e de cada disciplina, comunicação entre os agentes e qualidade das informações obtidas a partir dos usos pretendidos com o modelo.

\section{Estudo de caso}

A seguir, serão apresentados os processos para desenvolvimento do Plano de Execução BIM (BEP) e dos documentos e arquivos utilizados para desenvolvimento do estudo. 


\subsection{Plano de Execução BIM}

Os documentos elaborados pela equipe foram o BEP e as diretrizes de modelagem, ambos indispensáveis e com funções distintas. A elaboração dos documentos foi direcionada para uma experiência de modelagem colaborativa. Sendo assim, foram adotados os seguintes pontos como estrutura para o BEP: (1) equipes de projeto, (2) usos BIM, (3) caderno de diretrizes de modelagem e (4) nível de desenvolvimento dos modelos, além dos dados anteriormente estabelecidos como a descrição tecnológica necessária.

\section{- $\quad$ Parte 01 - Equipe de modelagem e matriz de responsabilidade}

A equipe de trabalho foi composta por profissionais da mesma formação: arquitetura e urbanismo. O agente responsável pela modelagem da disciplina de arquitetura foi encarregado pelo gerenciamento BIM (Figura 2) e, por isso, além da responsabilidade de modelagem, desenvolveu também as configurações prévias como: organização de worksets, coordenadas compartilhadas, norte de projeto e, por fim, verificação da qualidade da informação.

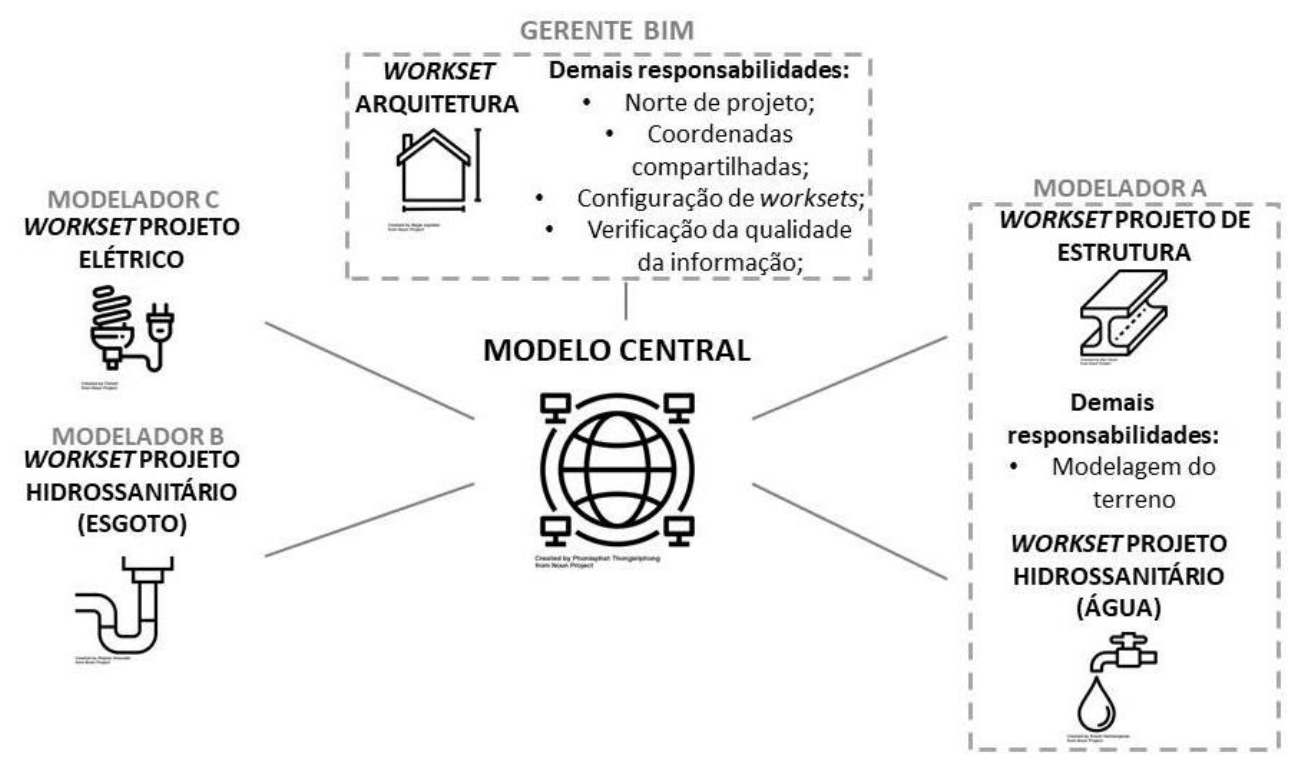

Figura 2: Organização do modelo central.

\section{- $\quad$ Parte 02 - Usos BIM pretendidos para os modelos}

Para definir os usos pretendidos do modelo, consultou-se a tabela de usos BIM desenvolvida pela Pennsylvania State University [6]. São eles: (1) condições existentes de modelagem, (2) estimativas de custo, (3) análise do sítio, (4) revisão de projetos, (5) coordenação 3D e (6) controle e planejamento do 3D. Após essa descrição de usos, ficou claro que somente a fase de operação de construções não deveria ser considerada no trabalho. Para essa fase ser contemplada em processos BIM, são necessárias informações mais específicas da construção (as-built) que não foram disponibilizadas pelo cliente.

\section{- $\quad$ Parte 03 - BIM Mandate ou caderno de diretrizes de modelagem para LOD 300}

O caderno de diretrizes de modelagem ou BIM Mandate foi elaborado para que as práticas de modelagem entre os agentes seguissem o padrão estabelecido, buscando garantir o Nível de Desenvolvimento no modelo final. Optou-se por um Nível de Desenvolvimento (LOD) 300, 
uma vez que o modelo permite o dimensionamento e execução do projeto e permite a extração de quantitativos de maneira direta ou indireta.

Após os estudos, listam-se na Tabela 1 os seguintes itens que estão presentes no BIM Mandate elaborado para a modelagem colaborativa:

Tabela 1: Itens presentes no BIM Mandate

\begin{tabular}{ll}
\hline Item & \multicolumn{1}{c}{ Descrição } \\
\hline Conceitos & Abordagem de conceitos preliminares. \\
$\begin{array}{l}\text { Nível de modelagem } \\
\text { por fase de projeto }\end{array}$ & $\begin{array}{l}\text { Fluxo de aprovação de projeto com o nível de maturidade dos } \\
\text { modelos. }\end{array}$ \\
$\begin{array}{l}\text { Orientações } \\
\text { preliminares de } \\
\text { modelagem }\end{array}$ & $\begin{array}{l}\text { Orientações sobre coordenadas compartilhadas, navegador de } \\
\text { projeto, sistema de unidades, sistema de coordenadas, níveis } \\
\text { utilizados nos modelos, orientações sobre o template da empresa etc. }\end{array}$ \\
$\begin{array}{l}\text { Diretrizes específicas } \\
\text { de modelagem (LOD }\end{array}$ & $\begin{array}{l}\text { Fichas para modelagem de cada elemento construtivo por disciplina: } \\
\text { arquitetura, estrutura, hidros sanitário e elétrico. Sendo explicitado } \\
\text { como ocorre a inserção e extração de informações. }\end{array}$ \\
\hline
\end{tabular}

\section{- Parte 04 - Desenvolvimento do template}

Entende-se por template, um arquivo previmente configurado para iniciar um trabalho em um software. No software utilizado nesse estudo de caso, o Autodesk Revit, o template possui a extensão .rte e pode ser inserido somente quando o usuário abre um novo arquivo de projeto. $\mathrm{O}$ template utilizado para o modelo citado no presente artigo foi desenvolvido para o mesmo, contendo informações e objetos BIM referentes às disciplinas abordadas (arquitetura, estrutura, hidrossanitário e elétrica). Ademais, o arquivo template - juntamente com as diretrizes de modelagem e o BEP - se mostrou essencial para o desenvolvimento do modelo de maneira uniformizada por todas as integrantes da equipe.

Por se tratar de um software BIM para a criação de modelos autorais, foi possível personalizar o espaço de trabalho em diversos níveis como: (1) unidades de medidas, (2) objetos inteligentes parametrizados e personalizados, (3) configurações de visualização e documentação, como cotas, textos e hachuras, (4) materiais e quaisquer informação a ser associada aos objetos BIM do modelo, entre outros [3].

\section{- $\quad$ Parte 05 - Estrutura dos worksets}

No Autodesk Revit, entende-se por Workset uma coleção de elementos em um projeto com compartilhamento de trabalho [12]. De modo geral, os worksets podem ser divididos como for mais conveniente aos coordenadores, existindo formas diversas de organização como: por pavimento, por partes do projeto, por sistema construtivo. No caso desta experiência, a divisão de worksets utilizada foi por disciplina trabalhada. Portanto, os worksets foram decompostos em: (1) arquitetura, (2) estrutura, (3) instalações de água fria, (4) instalações de esgoto e (5) elétrica. Além dos worksets supracitados, foram configurados também worksets auxiliares, como o os de eixos e o de terreno. 


\subsection{Desenvolvimento da modelagem}

O desenvolvimento do modelo contou com quatro encontros presenciais, com duração de $2 \mathrm{~h}$ $30 \mathrm{~min}$ e com todos os agentes envolvidos. No primeiro encontro, o norte de projeto, os worksets e as coordenadas compartilhadas foram configurados pelo gerente BIM. Além disso, foram criados respectivamente o modelo central e os modelos locais. Ainda no primeiro encontro foi dado início à modelagem das disciplinas de arquitetura e estrutura (Figura 4a) pelos modeladores responsáveis.

Posteriormente, no segundo encontro, o modelo estrutural foi concluído e os modelos de instalações (hidrossanitário e elétrico) foram iniciados (Figura 4b). Em relação à disciplina de arquitetura, foi dada continuidade à modelagem das paredes divisórias e iniciou-se a modelagem de revestimentos de paredes e pisos, assim como de elementos de forro, esquadrias e outros elementos construtivos pertencentes a esta disciplina. É importante salientar que alguns erros de projeto puderam ser corrigidos de forma quase que imediata devido à utilização de um modelo único e pela visualização de worksets possibilitada pelo software utilizado.
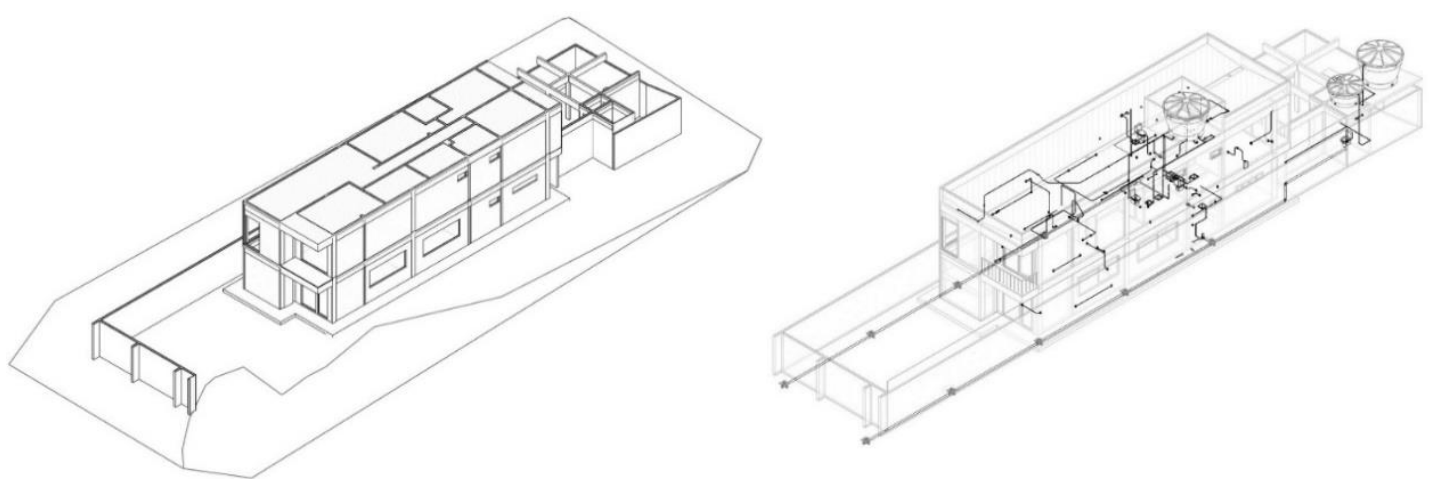

Figura 4a: Primeiro dia de modelagem e Figura 4b: Segundo dia de modelagem.

Após os dois primeiros dias de testes, foi evidenciada a necessidade das disciplinas de estrutura e arquitetura começarem com antecedência. Esse fato fica claro no momento em que observamos que a correta modelagem das tubulações hidrossanitárias depende da inserção prévia de peças sanitárias. No terceiro encontro, foi concluída a modelagem da arquitetura e foi dada continuidade à modelagem das instalações elétricas e hidrossanitárias, sendo estas concluídas no quarto encontro entre as profissionais.

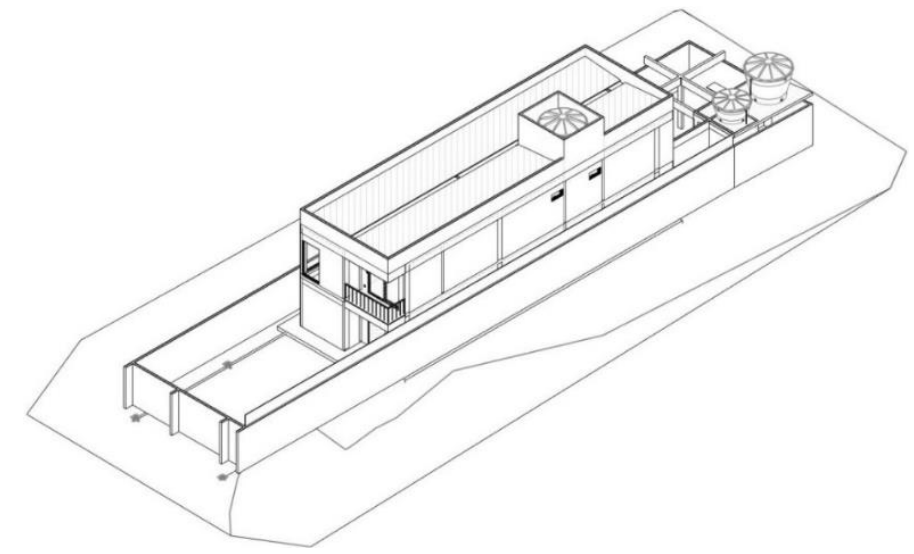

Figura 5: Modelo final. 


\section{Resultados}

A experiência de modelagem colaborativa deixou evidente que algumas disciplinas demandam mais tempo que outras (Tabela 2). Devido à quantidade de informação e à relação de dependência por parte das outras disciplinas, a modelagem de arquitetura mobilizou mais esforços por parte do profissional enquanto que o desenvolvimento do modelo de estrutura foi o mais célere, considerando uma estrutura de pórtico de concreto moldado no local. Um ponto de dificuldade em relação a modelagem de instalações é a pouca disponibilidade de objetos inteligentes tão eficientes como os de arquitetura e estrutura em repositórios abertos, o que acarretou a necessidade de mais tempo investido na configurações dos mesmos.

Tabela 2: Tempo de modelagem das disciplinas

\begin{tabular}{lc}
\hline \multicolumn{1}{c}{ Projeto } & Horas demandadas \\
\hline Projeto de arquitetura & $12 \mathrm{~h}$ \\
Projeto de estrutura & $3 \mathrm{~h}$ \\
Projeto Hidrossanitário - Água & $9 \mathrm{~h}$ \\
Projeto Hidrossanitário - Esgoto & $9 \mathrm{~h}$ \\
Projeto elétrico & $9 \mathrm{~h}$ \\
\hline
\end{tabular}

Outro fator analisado foi a comunicação entre os agentes. A frequência da comunicação entre os modeladores de cada disciplina mostra quais delas se interrelacionam mais entre si e, por isso, merecem mais atenção no momento do clash detection. A análise da frequência dessa comunicação (Tabela 3) também poderá compor uma lista de grupos de verificação (Tabela 4) de modelos para posterior auditoria do trabalho de modelagem realizado, uma das formas de garantir a qualidade da informação. Dessa forma, em trabalhos de modelagem colaborativa, é possível uma retroalimentação dos processos como forma de aprimoramento da equipe. Ficou evidente também como a disciplina de arquitetura tem papel protagonista na coordenação da modelagem. 
Tabela 3: Frequência de comunicação entre os modeladores

\begin{tabular}{|c|c|c|c|c|}
\hline & Disciplinas & $\begin{array}{c}\text { Tipo de } \\
\text { comunicação }\end{array}$ & Conteúdo & Recorrência \\
\hline Caso 1 & $\begin{array}{c}\text { Projeto elétrico e } \\
\text { projeto arquitetônico }\end{array}$ & Solicitação & $\begin{array}{c}\text { Modelagem dos forros } \\
\text { para auxiliar modelagem } \\
\text { dos conduíte que passam } \\
\text { no entreforro }\end{array}$ & 1 \\
\hline Caso 2 & $\begin{array}{c}\text { Projeto elétrico e } \\
\text { projeto arquitetônico }\end{array}$ & Compatibilização & $\begin{array}{l}\text { Solicitação de } \\
\text { compatibilização entre } \\
\text { forro e viga }\end{array}$ & 1 \\
\hline Caso 3 & $\begin{array}{c}\text { Projeto elétrico e } \\
\text { projeto arquitetônico }\end{array}$ & Aviso & Altura incorreta dos forros & 1 \\
\hline Caso 4 & $\begin{array}{c}\text { Projeto estrutural e } \\
\text { projeto arquitetônico }\end{array}$ & Solicitação & $\begin{array}{l}\text { Solicitação de } \\
\text { sincronização }\end{array}$ & 8 \\
\hline Caso 5 & $\begin{array}{c}\text { Projeto estrutural e } \\
\text { projeto hidrossanitário } \\
\text { de esgoto }\end{array}$ & Solicitação & $\begin{array}{l}\text { Solicitação de } \\
\text { sincronização }\end{array}$ & 1 \\
\hline Caso 6 & $\begin{array}{l}\text { Projeto arquitetônico e } \\
\text { projeto estrutural }\end{array}$ & Solicitação & $\begin{array}{l}\text { Solicitação para mover as } \\
\text { paredes de núcleo para } \\
\text { inserir as paredes de } \\
\text { revestimento }\end{array}$ & 1 \\
\hline Caso 7 & $\begin{array}{l}\text { Projeto arquitetônico e } \\
\text { projeto estrutural }\end{array}$ & Compatibilização & $\begin{array}{c}\text { Solicitação para mover } \\
\text { um dos pilares para fins } \\
\text { de compatibilização }\end{array}$ & 1 \\
\hline Caso 8 & $\begin{array}{l}\text { Projeto arquitetônico e } \\
\text { projeto elétrico }\end{array}$ & Compatibilização & $\begin{array}{c}\text { Solicitação para mover o } \\
\text { interruptor que estava } \\
\text { sobreposto ao pilar }\end{array}$ & 1 \\
\hline Caso 9 & $\begin{array}{l}\text { Projeto arquitetônico e } \\
\text { projeto elétrico }\end{array}$ & Compatibilização & $\begin{array}{c}\text { Solicitação de mudança de } \\
\text { altura de tomadas na } \\
\text { cozinha }\end{array}$ & 2 \\
\hline Caso 10 & $\begin{array}{c}\text { Projeto arquitetônico e } \\
\text { projeto hidrossanitário } \\
\text { de esgoto }\end{array}$ & Solicitação & $\begin{array}{c}\text { Solicitação para } \\
\text { modelagem de uma caixa } \\
\text { para hospedagem para a } \\
\text { caixa de passagem }\end{array}$ & 1 \\
\hline Caso 11 & $\begin{array}{c}\text { Projeto hidrossanitário } \\
\text { de esgoto e projeto } \\
\text { arquitetônico }\end{array}$ & Solicitação & $\begin{array}{l}\text { Solicitação de } \\
\text { sincronização }\end{array}$ & 2 \\
\hline Caso 12 & $\begin{array}{c}\text { Projeto hidrossanitário } \\
\text { de esgoto e projeto } \\
\text { estrutural }\end{array}$ & Compatibilização & $\begin{array}{c}\text { Solicitação de } \\
\text { compatibilização entre o } \\
\text { radier as caixas de } \\
\text { passagem }\end{array}$ & 1 \\
\hline
\end{tabular}


Tabela 4: Lista de grupos para verificação

\begin{tabular}{lcc}
\hline & Disciplinas & Elemento para os clashes \\
\hline Grupo 1 & Arquitetura e elétrica & Forro e vigas \\
Grupo 2 & Estrutura e elétrica & Pilares e tomadas \\
Grupo 3 & Estrutura e elétrica & Pilares e interruptores \\
Grupo 4 & Estrutura e arquitetura & Paredes e pilares \\
Grupo 5 & Estrutura e hidrossanitário de esgoto & Radier e caixas de passagem \\
\hline
\end{tabular}

As diretrizes de modelagem foram postas à prova ao final desta análise no que tange a qualidade das informações obtidas através do modelo. Para confirmar a validade dos quantitativos obtidos foram comparados aqueles extraídos do modelo e aqueles obtidos através do levantamento tradicional. Ao gerar as tabelas de quantificação de elementos construtivos, foi possível comprovar que componentes, como paredes, foram todos modelados de acordo com os critérios estabelecidos previamente e que, como consequência, o output obtido (área e comprimento) apresentava um reduzido desvio percentual (Tabela 5), de forma que se confirma assim a validade do processo proposto.

Tabela 5: Comparação entre os quantitativos de alvernaria extraídos do modelo x método manual

\begin{tabular}{cccc}
\hline & Modelo & Manual & Desvio percentual \\
\hline Área & $775,00 \mathrm{~m}^{2}$ & $779,44 \mathrm{~m}^{2}$ & $0,57 \%$ \\
Comprimento & $331,74 \mathrm{~m}$ & $324,66 \mathrm{~m}$ & $2,13 \%$ \\
\hline
\end{tabular}

\section{Conclusão}

A modelagem utilizando os worksets (ferramenta de trabalho colaborativo da Autodesk Revit) permitiu que os modeladores corrigissem incompatibilidades antes que o projeto passasse para a fase de documentação, evitando revisões e se mostrando uma metodologia que possibilita a redução de tempo, de falhas de projeto e da fase de compatibilização. Entretanto, há ainda alguns desafios ao trabalhar de maneira colaborativa. Entre os desafios, tem-se a rede de computadores que precisa ser previamente pensada para este fim. Outro ponto a ser vencido é dificuldade para modelagem colaborativa que tem reflexo na fragmentação dos processos de projeto na indústria da construção civil, o que dificulta a composição de uma equipe multidisciplinar para realização deste tipo de trabalho.

Outra questão a ser pontuada é o tempo e sequência necessários para as modelagens. $\mathrm{O}$ responsável pela modelagem de arquitetura precisa ser o precursor, começando a atuar antes do início da modelagem das outras disciplinas, no entanto, percebeu-se a necessidade de a modelagem da estrutura começar quase que concomitantemente à disciplina de arquitetura. Caso a modelagem estrutural comece antes, é interessante que se modele a infraestrutura completa para então ser iniciada a modelagem da disciplina de arquitetura. A disciplina de 
arquitetura é modelada ao longo de todo o processo, devido, principalmente, à grande quantidade de elementos e informações necessárias que precisam ser inseridas nos modelos. A experiência de análise da comunicação entre os modeladores mostrou como o modelador de arquitetura é naturalmente um agente coordenador e de tomada de decisão nos processos de modelagem. Foi evidenciado também que diferença de tempo de início entre uma disciplina e outra depende do porte do projeto e da experiência dos modeladores.

A falta de objetos BIM de instalações, elétricas e hidrossanitárias, que respondam ao modelo tão bem como os referentes à arquitetura e estrutura se torna uma questão relevante quando se propõe modelar todos os projetos de uma edificação. Os objetos referentes às instalações ainda contêm erros de parametrização, problemas de representação gráfica e são escassos quando comparados aos de arquitetura. Essa escassez deixa claro uma necessidade de investimento das empresas fabricantes de instalações em desenvolvimento de objetos inteligentes.

A análise das etapas que compõe o processo de modelagem colaborativa em BIM traz à reflexão seus entraves e potencialidades. Embora o método seja conhecido, fazer as reflexões aqui apresentadas é incompatível com o exercício projetual, quando existem cronogramas de entrega. Enumerar as etapas de concepção da estrutura de uma modelagem colaborativa em BIM, salientar o tempo e a ordem das modelagens entre disciplinas, compor de uma matriz de clash detection advinda das solicitações de esclarecimentos entre os atores do projeto são questões trazidas pelo trabalho que agregam à indústria da construção civil sempre em busca de uma maior assertividade em projetos.

\section{Referências}

[1] Amor, R. and R. Owen, "Beyond BIM - It's Not the End of the Road!," AECBytes, no. 58, Apr. 2011

[2] Catelani, W.S., Coletânea Implementação do BIM Para Construtoras e Incorporadoras. Volume 2 - Implementação BIM, Brasília, 2016.

[3] Catelani, W.S., Coletânea Implementação do BIM Para Construtoras e Incorporadoras. Volume 3 - Colaboração e Integração BIM, Brasília, 2016.

[4] Coelho, S.S. and C.C. Novaes, "Modelagem de Informações para Construção (BIM) e ambientes colaborativos para gestão de projetos na construção civil," Anais do VIII Workshop Nacional de Gestão do Processo de Projeto na Construção de Edifícios, Apr. 2008.

[5] Eastman, C.M., P. Teicholz, R. Sacks, and K. Liston, BIM handbook : a guide to building information modeling for owners, managers, designers, engineers, and contractors. Hoboken, N.J.: Wiley, 2008.

[6] Kreider R.G., and J. I. Messner, "The Uses of BIM: Classifying and Selecting BIM Uses," Pennsylvania State Univ., pp. 0-22, 2013.

[7] Manzione, L., "Proposição de uma estrutura conceitual de gestão do processo de projeto colaborativo com o uso do BIM." Tese de Doutoramento, Universidade de São Paulo, 2013.

[8] Messner, N., J., Anumba, C., Dubler, C., Goddman, S., Kasprzak, C., Kreider, R., Leicht, R., Saluja, C. and Zikic, "Building Information Modeling Execution Planning Guide Version 2.0," Comput. Integr. Constr. Res. Progr., 2010. 
[9] Santos, E.T., "BIM - Building Information Modeling: um salto para a modernidade na Tecnologia da Informação aplicada à Construção Civil”, Criação, Representação $e$ Visualização Digitais: tecnologias digitais de criação, representação e visualização no processo de projeto, Apr. 2012.

[10] Singh, V., N. Gu, and X. Wang, "A theoretical framework of a BIM-based multidisciplinary collaboration platform," Autom. Constr., vol. 20, no. 2, pp. 134-144, 2011, DOI: 10.1016/j.autcon.2010.09.011.

[11] Yin, R. K., Estudo de Caso: Planejamento e Métodos, 5 ed., Porto Alegre: Bookman, 2015.

[12] Knowledge.autodesk.com, Sobre os worksets, 2019. [Online]. Disponível em:

https://knowledge.autodesk.com/pt-br/guidref/RVT/2021/learn-explore/GUID-868102936510-4F5A-8009-27B4767CA136/REVITPRODUCTS. [Acessado: 16-jul-2019]. 